Besonders ausführlich wird das Präkambrium des atlantischen Bereichs dargestellt, in dem die Erforschung am weitesten fortgeschritten ist. Aus Zentralbrasilien und dem Guayana-Schild liegen nur wenige Untersuchungen vor. In den beiden folgenden Abschnitten wird die Stratigraphie und Paläogeographie des Paläozoikums und Mesozoikums geschildert, wobei sich der Verfasser auf seine eigenen Untersuchungen und vor allem auf das sehr umfangteiche Material der staatlichen Ölgesellschaft Petrobras stützen kann. Besonders interessant ist die Darstellung der allmählichen Öffnung des südlichen Atlantik.

Das vierte Hauptkapitel befaßt sich vorwiegend mit der geomorphologischen Ausgestaltung im Tertiär und Quartär. Hier wird die jugendliche Reliefbelebung Brasiliens zum erstenmal zusammenfassend behandelt. In den abschließenden Kapiteln folgten eine kurze Beschreibung der wichtigsten Lagerstätten und ausführliche englische und portugiesische Zusammenfassungen.

Das besondere Interesse BeurLeN's gilt der Entwicklung seit dem jüngeren Mesozoikum, die im Zusammenhang mit der vermutlichen Trennung von Afrika zur Bildung mächtiger Kreidefolgen im Küstenbereich und zu einer völligen Umgestaltung des hydrographischen Netzes geführt hat. Hier werden umfangreiche neue Beobachtungen mitgeteilt, die für den Mechanismus von Driftbewegungen viele Anregungen geben.

Die klare Darstellung, exläutert durch zahlreiche Abbildungen, macht das Buch zu einem wichtigen Nachschlagewerk für einen weiten Leserkreis. Insbesondere dürfte es von den Erdwissenschaftlern begrüßt werden, die in Südamerika arbeiten, und von denen, die sich mit Fragen der Kontinentalverschiebung beschäftigen. Um auch in Brasilien einen größeren $\mathrm{Kreis} z u$ erfassen, sollte unbedingt eine portugiesische Ubersetzung folgen.

R. PFLug (Heidelberg)

\title{
Errata
}

Rectification: En tête de l'article intitulé «Etude Géologique préliminaire des dépôts potassifères d'Iran» parue dans Vol. 5, No 3, au lieu de:

\section{W. Chazan}

Bureau des Recherches Géologiques et Minières, Orléans, France

lire: W. ChazAN

Direction des Mines, Ministère du Développement Industriel et Scientifique, Paris, France 